\title{
The ORC Patient/Tumor Classification-A New Approach: A New Challenge with Special Consideration for the Lung
}

\author{
Raymond Andrew Dieter Jr. ${ }^{1,2,3}$, George Kuzycz ${ }^{2,3}$, Raymond Andrew Dieter III ${ }^{4}$, Robert Sean Dieter ${ }^{5}$ \\ ${ }^{1}$ Past President International College of Surgeons, Chicago, USA; ${ }^{2}$ Center for Surgery LLC, Naperville, USA; ${ }^{3}$ Thoracic Surgeon, \\ DuPage Medical Group, Glen Ellyn, USA; ${ }^{4}$ Department of Cardiothoracic Surgery, University of Tennessee, Knoxville, USA; \\ ${ }^{5}$ Department Cardiology/Interventional, Loyola University Stritch School of Medicine, Maywood, USA. \\ Email: lnickerson@centerforsurgery.com
}

Received December 23 ${ }^{\text {rd }}$, 2011; revised April 14 ${ }^{\text {th }}$, 2011; accepted April 22 ${ }^{\text {nd }}, 2011$.

\begin{abstract}
Purpose: The development of malignancy is a life changing concern for many individuals. The classification of the tumor alone does not adequately take into consideration the patient's physical condition. Thus, a system to classify both the patient and the tumor has been followed-the ORC system. Method: Additional information regarding the patient and their health has been followed by most physicians but not systematically categorized. By using the individuals health information in addition to the TNM classification one can more adequately advise the patient. Thus O-operability, $R$-resectability, and C-curability are all considered and more appropriately define the patient and his/her tumor condition. Results: The patient's physical condition must be acceptable for the treatment-whether surgical or nonsurgical. Pulmonary, cardiac, muscular, renal or other disease entities must not be so severe as to prevent treatment (operability). The lesion should be in a location and of a size to afford possible excision-resectability, and the tumor should be potentially curable in order to justify major intervention. Thus, by combining the patient's specific health status as well as the tumor characteristics (TNM) a better clarification of the treatment, the options, and the prognosis are delineated. Conclusion: When a patient is seen with a tumor-malignant or benign, therapeutic considerations must include the individual's health status as well as the tumor prior to determining the treatment. Therefore, a system to consider both the health and the tumor is proposed-the ORC system.
\end{abstract}

Keywords: Patient Classification, Tumor Classification, Operability, Resectability, Curability, TNM

\section{Introduction}

Each year a large number of patients are seen with thoracic tumors. Most of these patients are afflicted with tumors of the lung and in particular with carcinoma of the lung. The patients are seen by their primary and consulting physicians and are classified according to their tumor in many instances. The current most frequently used tumor classification is the TNM classification. This classification is based on the tumor size, the nodal assessment and the metastatic condition of the patient. However, the TNM classification process does not take the patient's physical condition into consideration-only the tumor considerations. Therefore, prognosis and statistics may be skewed as a result of the condition of the patient even when they theoretically may have potentially curable lesions. For a number of years, we have reviewed and considered the patients from another non TNM standpoint utilizing a process or classification entitled the ORC classification. This classification takes the patient's physical condition into consideration when reviewing treatment options. In addition, the TNM program may be utilized as a portion of this patient/tumor evaluation. More recently, a number of articles have been published suggesting that there are potential problems related to TNM staging and suggesting modification of the system $[1,2]$.

\section{Concerns}

When one reviews the TNM classification, the patient's physical condition is only considered with respect to the tumor. Thus, a tumor may be small and meet the T1 criteria or it may be large and meet the criteria for a T4 lesion. The patient may further have no nodes involved or 
the lymphatic system may be extensively involved along with distant metastases. The TNM classification does not directly consider the patient's health. A classification which might consider both the patient and the tumor could assist in reviewing and understanding, to a greater degree, the potential for survival and treatment modalities in a patient afflicted with a thoracic malignancy.

Classification of the patient as well as classification of the tumor allows individuals from different areas to compare their results with a treater across the country or around the world. This assists one in understanding and developing the best treatment protocols for the patient and his/her disease. Whether these treatments are radiation, chemotherapy, surgical, radiofrequency or other programs, the optimum treatment as well as the prognosis may be better defined using a patient oriented system. Certainly since the International Union against Cancer (UICC) TNM classification was developed, we have seen a change in both the diagnostic as well as the therapeutic techniques available to a patient. Thus, modern clinical staging should involve consideration of the latest diagnostic techniques available including transbronchial ultrasound, transbronchial as well as transthoracic needle diagnostic techniques. Positron emission tomography, computed tomography scanning, magnetic resonance scanning, biochemical and molecular studies may be reviewed. This then may assist one in determining the pathologic staging of the disease as far as the TNM classification. Again, however, this does not define the patient and his/her ability to undergo surgical or interventional therapy. The current international staging system nicely defines the primary tumor, the nodal involvement and the metastatic situation. Staging of the tumor has been an attempt to define the opportunity for treatment, but only with regard to the tumor.

Unfortunately, this classification does not assess the patient's total medical condition and thus the operability or therapeutic viability of the patient and his/her potential surgical curability. The ORC concept evaluates the patient from a different and clinical aspect rather than from the TNM tumor classification. Thus, the OPERABILITY (O), RESECTABILITY (R) and CURABILITY (C) (ORC) program has been utilized clinically for evaluating a patient for years by physicians. Most physicians however, when utilizing this concept have not placed their evaluations in an organized fashion such as the TNM or ORC program for future comparative simplifications.

\section{Considerations}

The patient's operability must include his or her general physical condition. This would encompass the cardiac status, whether there has been a history of congestive heart failure, coronary artery disease, valvular disease or cardiomyopathy. Certainly knowledge of the pulmonary status of the patient and an adequate FEV1 or vital capacity, should be well documented. It is well known that an FEV1 of less than one is a poor prognosticator for thoracic surgery patients in whom the hospital mortality may be as much as $20 \%$ and few five-year survivors will occur. If the FEV1 is greater than 1 , however, the survival may be as much as $34 \%$ in selected T1N0M0 patients at five years. This thus demonstrates the need for classification of the patient as well as the tumor. Another consideration would be the age of the patient. Certainly elderly individuals in their 90's and 100's would on average have a greater risk for thoracic surgery and a shorter life span than an individual in their 50's or 60's. Other conditions including diabetes, other malignancies, muscle conditions, and their physiologic conditioning are important. The number of medications and dosage or whether the patient is a transplant patient will affect results. All of these concerns would effect the individual and his/her treatment-operability (O) and potential cure. The patients may thus have a TNM classification and tumor staging which would suggest a favorable situation, but his/her general condition would not be so appropriate.

Resectability (R) considers the size and location of the tumor. The lesion may be very large and still be resectable. In other situations the tumor may be smaller but in a location rendering it unresectable. Preoperative knowledge of the cell type may also be considered as to resectability-particularly with reference to the small or oat cell tumors. In addition, at the time of surgery, in our experience, a hard or firm tumor is much more readily resected for potential cure than a soft tumor. A soft "mushy" tumor is more readily entered at surgery and as a result more difficult to dissect. In addition, PET scanning as well as the TNM classification would have a place in determining resectability.

Lastly, curability (C) would take into account whether there is any spread of the tumor and any potential exceptions. Thus, a tumor that has spread to the brain as a solitary lesion may be potentially resectable and potentially curable, particularly when it is a non-small cell lesion. Adrenal metastasis also may be considered in this area of distant lesions, as well as the extension of the tumor locally (ex. to the ribs), and thus whether it is potentially curable and if it is resectable. Certainly in the future biochemical and molecular determinants will play a larger role in patient and tumor evaluation. If one then contemplates the ORC classification of the patient (operable, resectable and curable), the ORC grouping would add value to the understanding of the patient and their potential results from treatment of their tumor. The ORC program would evaluate both the whole patient as well as 
the tumor. The TNM program primarily considers the tumor, in the clinical, or pathologic staging situation whereas the entire patient and the tumor directs the ORC program.

\section{Surgical Classification of Patients}

The ORC system (Table 1) utilizes factors involving the patient's general health and the tumor in making surgical recommendations. The operable patients may be classified as O1 (low risk, should survive), O2 (medium risk, a life threatening procedure but potential to survive) and O3 (high risk surgical procedure with a high chance for a demise). Resectability (R) may be evaluated by the physician and categorized as R1 (usually resectable), R2 (probably resectable), and R3 (little chance of resection). Curability (C) again is separated into C1 (should be curable), C2 (50-50 chance of cure), C3 (probably not curable). Thus if one has an O1 R1 C1 classification, the chances of curability with survivability are much higher in these individuals than if they have an $\mathrm{O} 3 \mathrm{R} 3 \mathrm{C} 3$ patient and tumor classification. Table 2 lists potential classifications of the ORC program and defines who may or may not require surgery. Table 3 provides a staging of these patients according to the ORC program and further defines who may potentially be operable or not operable. Certainly the Stage IV (O3 R3 C3) patient would only be considered for surgery as an emergency life-saving procedure that one might see with massive hemorrhage or in similar urgent palliative situations where cure or palliation would seldom be of value. You would offer surgery to all O1 R1 C1 patients and seldom operate the patients with an O3 R3 C3 classification. A comparative tumor

Table 1. The ORC system.

\begin{tabular}{lll}
\hline \multirow{2}{*}{ Operability } & 1) & O1 = low risk-should survive \\
& 2) & O2 = medium risk-life threatening \\
3) & O3 = high risk-great chance for demise \\
Resectability- & 1) & R1 = high probability \\
(correlate with TNM) & 2) & R2 = medium probability \\
& 3) & R3 = low probability \\
1) & C1 = high chance of cure \\
Curability & 2) & C2 = average chance to cure \\
& 3) & C3 = low chance to cure \\
\hline
\end{tabular}

Table 2. ORC classification system.

\begin{tabular}{lll}
\hline & Class & \\
\hline O1 R1 C1 & O2 R1 C1 & O3 R1 C1* \\
O1 R1 C2 & O2 R1 C2 & O3 R1 C2* \\
O1 R1 C3* & O2 R1 C3* & O3 R1 C3* \\
O1 R2 C1 & O2 R2 C1 & O3 R2 C1* \\
O1 R2 C2 & O2 R2 C2 & O3 R2 C2* \\
O1 R2 C3* & O2 R2 C3* & O3 R2 C3* \\
O1 R3 C1* & O2 R3 C1* & O3 R3 C1* \\
O1 R3 C2* & O2 R3 C2* & O3 R3 C2* \\
O1 R3 C3* & O2 R3 C3* & O3 R3 C3* \\
\hline
\end{tabular}

*No or seldom do surgery: any O3 R3 or C3.
Table 3. The ORC staging system and surgery.

\begin{tabular}{ccll}
\hline \multicolumn{2}{c}{ TNM Staging Concept } & $\begin{array}{l}\text { Surgery: ORC } \\
\text { Class }\end{array}$ & $\begin{array}{l}\text { No Surgery: } \\
\text { ORC Class }\end{array}$ \\
\hline \multirow{2}{*}{ a } & Stage IA & O1-2R1C1 & O3R1-2C1-2 \\
& Stage IB & O1-2R1-2C1-2 & O3RI-2C1-2 \\
b & Stage IIA & O1-2R1-2C1-2 & O3 \\
& Stage IIB & O1-2R1-2C1-2 & \\
c & Stage IIIA & O1-2R1-2C1-2 & O3 R3 C3 \\
& Stage IIIB & O1-2R1-2C1-2 & \\
d & Stage IV & $\begin{array}{l}\text { O1-2R1-2C1-2 } \\
\text { Emergency }\end{array}$ & O3 R3 C3 \\
\hline
\end{tabular}

classification with the TNM staging may then provide additional information for the patient and the treating physician.

\section{Discussion}

The staging system for non-small cell lung cancer utilizing the TNM program has led to numerous authors recommending consideration for modification or revamping of the TNM program. Lee, et al., discussed the significance of extranodal tumor extension and the staging system for these patients [1]. Barnes, in an editorial, postulated that it is time for an overhaul of the TNM system and stated that any staging system should accurately reflect the prognosis of the patient, and if it does not, it should be modified [2]. Any staging system should assist in developing further therapy needs and the recognition for such. The R2 C3 or R3 C3 individuals are examples of this class of patients. Kameyama, et al. have suggested that problems with the TNM staging program particularly involve those individuals with the Stage III non-small cell lung cancer [3]. Resectability as well as curability are important considerations in these patients. Paci, et al., in discussing controversies regarding the UICC-TNM classification, have suggested that a model for a useful diagnostic and therapeutic path for optimizing available resources should be developed, and that the current staging system does not meet these needs [4].

Evaluation of lung tumors should not be made separately from evaluation of the patient. Molecular biology, histochemistry, genetics, and other influences will be of increasing value in the future [5]. No system, the TNM nor the ORC system, will apply to all patients or conditions but whatever the system it should evaluate the patient as well as the tumor. Modifications and future knowledge will eventually direct further appropriate adjustments in classification for therapy, surgical or non-surgical, to benefit the patient and research [6]. The ORC concept is a step forward in tumor bearing patient classification for therapy [7].

\section{Conclusions}

1) Consideration of various concerns for the patient and 
their disease requires constant review of our classifications and techniques to avoid inappropriate therapy and surgery.

2) A proposal to revise the current classification techniques is presented which considers the patient as a whole and not primarily the tumor.

3) The ORC concept, which demonstrates the operability and potential survivability of patients with lung tumors, may be modified for other diseases and tumors.

4) Positive characteristics of the TNM program may be introduced into the ORC classification for further patient evaluation and treatment determinations.

\section{REFERENCES}

[1] Y.-C. Lee, C.-T. Wu, S.-W. Kuo, Y.-T. Tseng and Y.-L. Chang, "Significance of Extranodal Extension of Regional Lymph Nodes in Surgically Resected Non-Small Cell Lung Cancer,” Chest, Vol. 131, No. 4, April 2007, pp. 993-998. doi:10.1378/chest.06-1810

[2] D. J. Barnes, "The Staging System for Non-Small Cell Lung Cancer, Time for an Overhaul? (Editorial)," Chest, Vol. 131, No. 4, April 2007, pp. 948-949. doi:10.1378/chest.06-3126

[3] K. Kameyama, C. Huang, D. Liu, T. Okamoto, E. Haya- shi, Y. Wamota and H. Wokomise, "Problems Related to TNM Staging: Patients with Stage III Non-Small-Cell Lung Cancer,” Journal of Thoracic Cardiovascular Surgery, Vol. 124, No. 3, September 2002, pp. 503-510. doi:10.1067/mtc.2002.123810

[4] M. Paci, G. Sgarbi, G. Ferrari, S. DeFranco and V. Annessi, "Controversies over UICC-TNM Classification of Non-Small Cell Lung Cancer, Model for a Diagnostic Path (Letter to the Editor)," Chest, Vol. 122, No. 2, August 2002, p. 754.

[5] M. Chang, S. Mentzer, Y. Colson, P. Linden, M. Jaklitsch, S. Lipsitz and D. Sugarbaker, "Factors Predicting Poor Survival after Resection of Stage IA Non-Small-Cell Lung Cancer," Journal Thoracic and Cardiovascular Surgery, Vol. 134, October 2007, pp. 850-856. doi:10.1016/j.jtcvs.2007.03.044

[6] P. D. Kiernan, M. J. Sheridan, J. Lamberti, T. Lorusso, V. Hutrick, B. Vaughan and P. Grailing, "Late Stage (III and IV) Non-Small-Cell Lung Cancer, Results of Surgical Resection at Inova Fairfax Hospital,” Southern Medical Journal, Vol. 98, No. 11, November 2005, pp. 10881094. doi:10.1097/01.smj.0000177344.48950.65

[7] R. A. Dieter, G. Kuzycz and R. Dieter, "The ORC Concept,” Presentation: European Federation Meeting Int'l College Surgeons, Antalya, October 2007. 